\title{
The distance to IC 4499 from $K$-band photometry of 32 RR Lyrae stars ${ }^{\star}$
}

\begin{abstract}
J. Storm ${ }^{\star \star}$
Astrophysikalisches Institut Potsdam, An der Sternwarte 16, 14482 Potsdam, Germany

Received 8 September 2003 / Accepted 14 November 2003

Abstract. Single epoch $K$-band photometry of 32 RR Lyrae stars in the young galactic globular cluster IC 4499 is presented. The mean magnitudes have been estimated using the $K$-band template light-curves of Jones et al. (1996). We derive an independent estimate of the distance and reddening for the cluster based on the new $\left\langle M_{K}\right\rangle-\log P-[\mathrm{Fe} / \mathrm{H}]$ relation from Bono et al. (2003) which has a zero-point in agreement with the canonical LMC distance of $(m-M)_{0}=18.5$. For an assumed metallicity of $[\mathrm{Fe} / \mathrm{H}]=-1.65 \pm 0.1$ we find $(m-M)_{0}=16.47 \pm 0.04 \pm 0.06$ (random and systematic errors respectively) and $E(B-V)=0.24 \pm 0.03$ in good agreement with the findings of Walker \& Nemec (1996) based on optical data. The distance estimate is very robust to uncertainties in the reddening estimate but naturally depends directly on the adopted zero-point of the $\left\langle M_{K}\right\rangle-\log P-[\mathrm{Fe} / \mathrm{H}]$ relation.
\end{abstract}

Key words. stars: distances - stars: fundamental parameters - stars: horizontal-branch - stars: variables: RR Lyrae

\section{Introduction}

The globular cluster IC 4499 (RA: $15^{\mathrm{h}} 00^{\mathrm{m}} 19^{\mathrm{s}}$, Dec: $-82^{\circ} 12^{\prime} 50^{\prime \prime}$; 2000.0) is among the youngest galactic globular clusters (Ferraro et al. 1995; Fusi Pecci et al. 1995). It is of Oosterhoff type I and is thus likely to have zero or even retrograde rotation around the Galactic center (van den Bergh 1993; Lee \& Carney 1999). It is a fairly low density cluster with one of the highest numbers of RR Lyrae stars per unit luminosity (Suntzeff et al. 1991) containing about 100 RR Lyrae stars. These extreme characteristics suggests that the cluster might be a late addition to the Galaxy, which makes the cluster particularly interesting to study.

Optical color-magnitude diagrams have been presented by Sarajedini (1993) and Ferraro et al. (1995), the latter extending two magnitudes below the main-sequence turn-off. The RR Lyrae stars have been the subject of several investigations (Coutts et al. 1975; Clements et al. 1979, 1986; Walker \& Nemec 1996 (WN96 in the following)) since their discovery (Fourcade \& Laborde 1969; Fourcade et al. 1974). These investigations have revealed that the RR Lyrae stars span the full range of pulsation modes currently known, including stars exhibiting double mode pulsations, which can be used to determine the mass of these stars. WN96 has even suggested that some of the stars might pulsate in the second overtone. The range of the stellar masses has been shown by Sarajedini (1993) to be very limited as judged from the double mode pulsators.

The reddening towards IC 4499 is fairly high, $E(B-V)=$ $0.22 \pm 0.02$ (WN96) and thus a distance estimate which is

\footnotetext{
* Based on data from the European Southern Observatory.

$\star \star$ e-mail: jstorm@aip.de
}

insensitive to reddening will provide an important additional constraint on the distance to this cluster.

The $\left\langle M_{K}\right\rangle-\log P$ relation for RR Lyrae stars, pioneered by Longmore et al. (1986) and refined further by Longmore et al. (1990), is a very powerful tool for distance determination. The method is theoretically based on the pulsation equation from van Albada \& Baker (1971) which links the period to the luminosity of an object. Bono et al. (2001), and most recently Bono et al. (2003) (B03 hereinafter) has refined the method using pulsational stellar models to include a small but significant metallicity term, and we will use this calibration in the following. It has been shown by Butler (2003), through a comparison of inner and outer region RR Lyrae stars in M 3, that the method is insensitive to environmental effects. It is also rather insensitive to errors in both $[\mathrm{Fe} / \mathrm{H}]$ and reddening, and as the amplitudes of RR Lyrae stars in the $K$-band are smaller than about 0.3 mag it is feasible to apply the relation to single epoch data if a sufficiently large number of stars can be observed. Using recent ephemerides for the IC 4499 stars we can even phase the observations and use template light-curves to improve our estimate of the mean $K$-magnitudes.

\section{The data}

We used the IRAC-2B near-IR camera (Moorwood et al. 1992) mounted at the ESO-MPI $2.2 \mathrm{~m}$ telescope at La Silla on the night Sep. 17/18, 1995. The detector was a 256 by 256 pixel NICMOS-3 array which was read out using the so called "nondestructive read" mode. The lens LC, which provides a projected pixel size of 0.5 arcsec was employed, and the seeing was typically 1.3 arcsec FWHM. 
Two adjacent fields (labeled $\mathrm{A}$ and $\mathrm{B}$ in the following) including the center of IC 4499 were selected on the basis of the charts in Clement et al. (1986) to cover a large number of known RR Lyrae variables as well as a good range in period.

The observations were performed in a regular pattern of two dithered target frames interspersed by two dithered sky frames (several arc-minutes away) followed by the same sequence again, after offsetting the telescope by a few arcsec. Each frame was made of six $10 \mathrm{~s}$ integrations which were averaged on-line by the pre-processing system. For field A, 16 individual frames were obtained through the $K^{\prime}$ filter and for field B, 11 such frames were obtained giving a total of 16 and $11 \mathrm{~min}$, respectively, of on-target integration time. The mean HJD for the two datasets is 2449978.5198 and 2449978.5661 respectively.

\section{Data reduction}

\subsection{Pre-processing}

All the data were corrected for non-linearity using the ctio.irlincor command within IRAF $^{1}$. Polynomial coefficients of coeff $1=1.0$, coeff $2=3.808477 \times 10^{-2}$, coeff $3=$ $5.37237 \times 10^{-2}$ were adopted on the basis of the reported nonlinearity for the array (Lidman 1995).

Flatfields were produced as the difference between exposures of the white screen in the dome with the quartz lights on and off, respectively. The IRAC-2 camera is known to suffer from parasitic light in the flatfields at the 5\% level, and a standard star was mapped in a 4 by 4 grid across the array on the night of the IC 4499 observations as well as on the previous night in order to determine the necessary correction for the flat fields. The counts in each position were fitted by a two dimensional second order polynomial for each night. The results were in good agreement, to within $3 \%$, and the averaged correction was applied to the flat field frame.

Sky frames were computed as the average of four dithered sky exposures obtained before and after the science frame. The "avsigclip" algorithm of the IRAF imcombine command with a threshold value of $\sigma=5$ was employed to eliminate stars in the sky fields. For each target frame the corresponding sky frame was subtracted and bad pixels were removed by linear interpolation. The resulting targets frames were finally averaged to produce the final target frames.

\subsection{Standard star photometry}

Fourteen standard star measurements were performed on the night Oct. $1 / 2,1995$, to determine the color terms of the camera in the $J$ and $K^{\prime}$ filters and the airmass coefficients. Each star was observed 4 times with the star imaged in four different positions on the array. The stars were selected from the list of Carter \& Meadows (1995) to cover as large a range in $(J-K)$ as possible, i.e. from -0.02 to 0.96 , and they were observed at airmasses ranging from 1.03 to 1.80 . Using the conversion

\footnotetext{
1 IRAF is distributed by the National Optical Astronomical Observatories, which is operated by AURA, Inc., under cooperative agreement with the NSF.
}

from the Carter system to the CIT system determined by Carter (1993) we find:

$$
\begin{aligned}
K_{\mathrm{CIT}}= & K_{\text {inst }}^{\prime}+0.014( \pm 0.02) \times\left(J-K^{\prime}\right)_{\text {inst }} \\
& -0.17( \pm 0.03) \times X+\text { const }
\end{aligned}
$$

with an rms scatter of $0.024 \mathrm{mag}$. We thus find no significant color term, and will assume a zero effect in the following.

On the night of the IC 4499 observations we also observed the standard star HD 136879 (Carter \& Meadows 1995) before and after the observations of IC 4499 in a pattern of four positions on the detector array. The airmass for the standard star measurements was 1.44 and 2.13 respectively, while the airmass for the IC 4499 observations ranged from 1.85 to 2.05 (IC 4499 is a very southern object). The standard star measurements were interpolated in airmass to provide the appropriate offset for the IC 4499 data. Curves of growth were determined from artificial aperture photometry of the standard star and an aperture radius of 20 pixels (10 arcsec) was used to determine the offset to the standard system. From the rms scatter for Eq. (1) we estimate the error of the $K$-band zero-point to be $0.025 \mathrm{mag}$.

\subsection{Photometry}

The DAOPHOT package (Stetson 1987) within IRAF was used to find the stars within the averaged frames and for performing photometry on them by point spread function (PSF) fitting. Due to the sparse nature of IC 4499 crowding is not an issue for PSF fitting photometry.

The aperture corrections for the PSF photometry were determined from a number of well exposed and reasonably isolated stars in the IC 4499 fields. The aperture photometry was performed after all the stars, apart from the stars selected for offset determination, had been subtracted by the IRAF/DAOPHOT substar routine. The resulting aperture corrections for five stars in each field agreed to better than 0.02 mag.

The RR Lyrae stars were identified from the finding chart of WN96. The final magnitudes on the CIT system are tabulated in Table 1 together with the periods and the type of pulsation as determined by WN96. The pulsation type is indicated by "ab" for fundamental mode pulsators, "c" for first overtone, "d" for double mode, and "e?" for possible second overtone pulsators. The estimated errors provided by DAOPHOT have been tabulated as well. Note that the errors on the magnitudes for stars in field B are significantly larger than in field A due to the shorter integration time.

The observed magnitudes have been collected at a specific time as already mentioned in Sect. 2 so with an appropriate ephemeris for each star we can determine the phase of observation. Alistair Walker has kindly provided the full optical light curves from WN96 in machine readable format and using their period estimates we have determined the epoch of maximum $V$-light for each of the stars. On this basis we have determined the phase corresponding to the observed $K$-magnitude. We have then used the $K$-band template light curves from Jones et al. (1996) to compute the corresponding intensity averaged 
Table 1. Periods observed $K$ magnitudes and the estimated mean $K$ magnitudes for the RR Lyrae stars in the two fields. The fundamental period is listed for the RRd stars. The type of pulsator as determined by WN96 is listed as well. The last column indicates in which of the two fields the star was observed.

\begin{tabular}{rcccccc}
\hline \hline ID & $\log P$ & $K$ & $\sigma(K)$ & $\langle K\rangle$ & Type & Field \\
\hline 1 & -0.2147 & 15.92 & 0.06 & 15.82 & $\mathrm{ab}$ & $\mathrm{A}$ \\
4 & -0.2051 & 15.79 & 0.10 & 15.80 & $\mathrm{ab}$ & $\mathrm{B}$ \\
5 & -0.2542 & 15.85 & 0.10 & 15.89 & $\mathrm{ab}$ & $\mathrm{B}$ \\
11 & -0.1996 & 15.73 & 0.04 & 15.79 & $\mathrm{ab}$ & $\mathrm{A}$ \\
13 & -0.2911 & 16.00 & 0.05 & 16.08 & $\mathrm{ab}$ & $\mathrm{A}$ \\
14 & -0.3009 & 15.67 & 0.13 & 15.63 & $\mathrm{ab}$ & $\mathrm{B}$ \\
15 & -0.2242 & 15.99 & 0.12 & 16.04 & $\mathrm{ab}$ & $\mathrm{B}$ \\
17 & -0.3006 & 16.05 & 0.11 & 15.96 & $\mathrm{ab}$ & $\mathrm{B}$ \\
18 & -0.3248 & 16.22 & 0.14 & 16.22 & $\mathrm{~d}$ & $\mathrm{~B}$ \\
23 & -0.2944 & 16.16 & 0.07 & 16.08 & $\mathrm{ab}$ & $\mathrm{A}$ \\
24 & -0.2869 & 15.98 & 0.10 & 16.06 & $\mathrm{ab}$ & $\mathrm{B}$ \\
29 & -0.4405 & 16.25 & 0.08 & 16.24 & $\mathrm{c}$ & $\mathrm{A}$ \\
30 & -0.2766 & 15.97 & 0.05 & 15.99 & $\mathrm{ab}$ & $\mathrm{A}$ \\
32 & -0.4437 & 15.33 & 0.03 & 15.31 & $\mathrm{c}$ & $\mathrm{A}$ \\
37 & -0.2481 & 15.82 & 0.10 & 15.87 & $\mathrm{ab}$ & $\mathrm{B}$ \\
48 & -0.2842 & 16.23 & 0.08 & 16.05 & $\mathrm{ab}$ & $\mathrm{A}$ \\
49 & -0.3018 & 15.91 & 0.12 & 15.98 & $\mathrm{ab}$ & $\mathrm{B}$ \\
50 & -0.2474 & 15.84 & 0.06 & 15.92 & $\mathrm{ab}$ & $\mathrm{A}$ \\
51 & -0.3214 & 16.20 & 0.07 & 16.20 & $\mathrm{~d}$ & $\mathrm{~A}$ \\
55 & -0.4455 & 16.08 & 0.14 & 16.10 & $\mathrm{c}$ & $\mathrm{B}$ \\
56 & -0.5383 & 16.21 & 0.14 & 16.24 & $\mathrm{e} ?$ & $\mathrm{~B}$ \\
58 & -0.3005 & 15.92 & 0.12 & 15.88 & $\mathrm{ab}$ & $\mathrm{B}$ \\
59 & -0.3188 & 15.97 & 0.07 & 15.97 & $\mathrm{~d}$ & $\mathrm{~A}$ \\
70 & -0.2700 & 15.98 & 0.06 & 15.87 & $\mathrm{ab}$ & $\mathrm{A}$ \\
71 & -0.3045 & 15.96 & 0.11 & 15.96 & $\mathrm{~d}$ & $\mathrm{~B}$ \\
72 & -0.1666 & 15.78 & 0.09 & 15.80 & $\mathrm{ab}$ & $\mathrm{B}$ \\
81 & -0.4022 & 15.71 & 0.05 & 15.70 & $\mathrm{c}$ & $\mathrm{A}$ \\
82 & -0.2654 & 15.83 & 0.10 & 15.88 & $\mathrm{ab}$ & $\mathrm{B}$ \\
89 & -0.4493 & 16.14 & 0.14 & 16.14 & $\mathrm{c}$ & $\mathrm{B}$ \\
90 & -0.3113 & 16.07 & 0.13 & 16.07 & $\mathrm{~d}$ & $\mathrm{~B}$ \\
97 & -0.5402 & 16.14 & 0.14 & 16.16 & $\mathrm{e} ?$ & $\mathrm{~B}$ \\
1575 & -0.5534 & 16.44 & 0.09 & 16.41 & $\mathrm{e} ?$ & $\mathrm{~A}$ \\
\hline & & $\mathrm{mag}$ & $\mathrm{mag}$ & $\mathrm{mag}$ & & \\
\hline & & & & & &
\end{tabular}

mean $K$-magnitude, $\langle K\rangle$, based on the type of pulsation and the $V$-band amplitude as given by WN96. For the RRd stars we have not attempted a phase correction and for the putative RRe stars we have assumed that they are actually RRc stars as done by Castellani et al. (2003). These estimates of the mean $K$-magnitude have also been tabulated in Table 1 .

\section{Results}

\subsection{The $\left\langle M_{K}\right\rangle-\log P$ relation}

The $\left\langle M_{K}\right\rangle-\log P$ relation has been established by Longmore et al. (1990) who determined the slope from photometry of RR Lyrae stars in 8 different galactic globular clusters.
Recently, B03 has developed the relation further to incorporate a small but significant metallicity effect and they find the relation:

$$
\left\langle M_{K}\right\rangle=-0.770-2.101 \log P+0.231[\mathrm{Fe} / \mathrm{H}] .
$$

For a globular cluster like IC 4499 where we assume that there is no significant spread in $[\mathrm{Fe} / \mathrm{H}]$ among the RR Lyrae stars, we can simply insert the best estimate of the metallicity in Eq. (2) to get a $\left\langle M_{K}\right\rangle-\log P$ relation. WN96 discusses the available metallicity estimates for IC 4499 and find a value of $[\mathrm{Fe} / \mathrm{H}]=-1.65 \pm 0.1$ based on spectroscopic measurements of Ca II triplet lines in four RGB stars by Cannon (1992) and from a $(V,(V-I))$ color-magnitude diagram.

We insert this value in Eq. (2) and for each star we can then determine the distance modulus as:

$(m-M)_{K}=\langle K\rangle-\left\langle M_{K}\right\rangle=\langle K\rangle-1.151-2.101 \log P_{0}$.

The relation refers to the fundamental period and thus it is necessary to fundamentalize the periods of the higher order pulsators. We adopt the period ratios as used by WN96, i.e. $(\mathrm{P} 2: \mathrm{P} 1: \mathrm{P} 0)=(1.575: 1.3439: 1.0)$ and thus $\log P_{0}=\log P_{1}+$ 0.128 and $\log P_{0}=\log P_{2}+0.197$ for the RRc and RRe stars, respectively.

In Fig. 1 we have plotted the $\langle K\rangle$ magnitudes for the individual stars versus $\log P_{0}$, the fundamentalized period. Again we have assumed that the putative RRe stars are actually RRc stars. They actually fall exactly on the general relation. If we choose to fundamentalize the period of these stars as second overtone stars they would appear where the open diamonds in Fig. 1 have been plotted which gives a less satisfying match.

Inspecting Fig. 1 further, we see that three of the stars, marked with open symbols (V14, V32 and V81), are overly bright for their period when compared to the bulk of the stars. WN96 also found both V32 and V81 to be over-luminous in the optical, and they suspected both stars to have an unresolved red companion.

The detection limit is fainter than the faintest RR Lyrae star by about $1 \mathrm{mag}$ in the shallow field (B) so the sample is complete and can not suffer from Malmquist bias.

\subsection{The reddening and the distance modulus}

For each star we can now use Eq. (3) to determine the distance modulus and we can then compute the weighted mean of the resulting distances. If we use the sample of $29 \mathrm{RRab}, \mathrm{RRc}$, and $\mathrm{RRd}$ stars excluding the three outliers we find $(m-M)_{K}=$ $16.555 \pm 0.016$ with $\sigma=0.087 \mathrm{mag}$. If we include V14 and V32 we find $(m-M)_{K}=16.526 \pm 0.023$ with $\sigma=$ $0.12 \mathrm{mag}$, which is only slightly smaller than the previous result. The three possible RRe stars do not affect the distance estimate significantly, independently of how we choose to fundamentalize them. Finally, if we choose to simply use the random phase $K$ magnitudes as a proxy for the mean $K$-magnitude instead of the template approach described above, we find $(m-M)_{K}=16.553 \pm 0.019$ with $\sigma=0.104$, basically an identical result but with a slightly larger uncertainty.

Due to the relative insensitivity of the $\left\langle M_{K}\right\rangle-\log P$ relation to reddening, we can determine an independent value for 


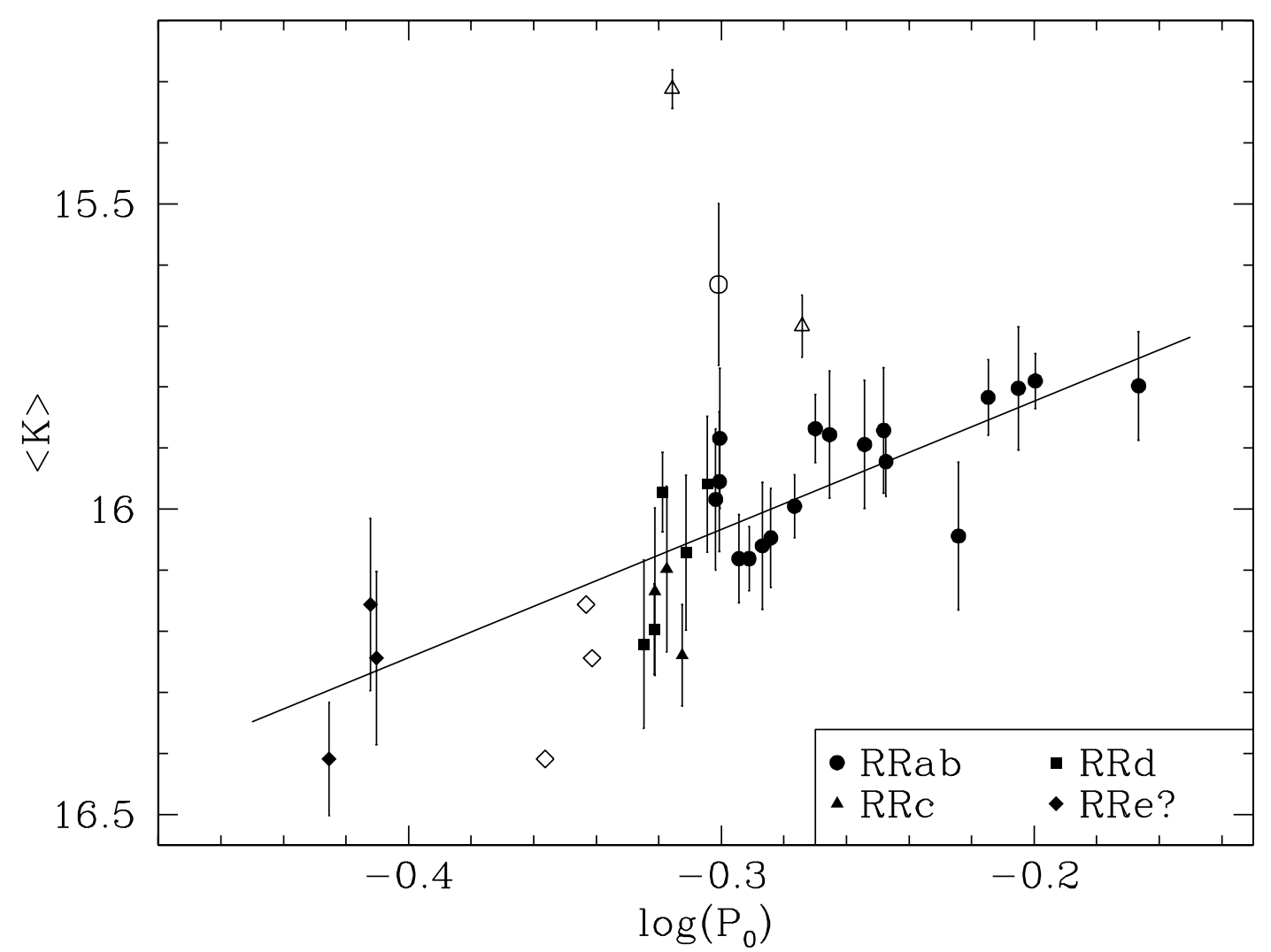

Fig. 1. The $\langle K\rangle$ magnitudes plotted against fundamental period. For the RRd stars the single epoch $K$ is plotted. The open symbols were not included in the fit. The filled diamonds labeled RRe? represents the putative RRe stars from WN96 but fundamentalized as RRc stars. The open diamonds show where these stars would appear had they been fundamentalized as second overtone pulsators. The line shows Eq. (2) for $[\mathrm{Fe} / \mathrm{H}]=-1.65$ and shifted by our best distance estimate of $(m-M)_{K}=16.55$.

the reddening towards IC 4499 from the mean $V$-magnitude of the RR Lyrae stars as determined by WN96 and the $\left\langle M_{V}\right\rangle-[\mathrm{Fe} / \mathrm{H}]$ relation. For consistency we use the calibrations presented by B03 for both relations, and thus in addition to Eq. (2) we adopt:

$$
\left\langle M_{V}\right\rangle=0.177( \pm 0.07)[\mathrm{Fe} / \mathrm{H}]+0.718( \pm 0.07)
$$

which is valid for metal-poor stars $([\mathrm{Fe} / \mathrm{H}]<-1.6)$.

WN96 find the mean $V$-magnitudes of the RR Lyrae stars to be $<V_{\mathrm{RR}}>=17.652 \pm 0.006$ and thus $(m-M)_{V}=17.226 \pm$ 0.07. Adopting $A_{V}=3.1 \times E(B-V)$ and $A_{K}=0.11 \times A_{V}$ (Cardelli et al. 1989) and using the constraint that the reddening corrected distance estimates from both the $\left\langle M_{K}\right\rangle-\log P$ and $\left\langle M_{V}\right\rangle-[\mathrm{Fe} / \mathrm{H}]$ relations should be the same, we can write the relation:

$(m-M)_{V}-A_{V}=(m-M)_{K}-A_{K}$

from which follows:

$A_{V}=\left[(m-M)_{V}-(m-M)_{K}\right] / 0.89$

$A_{V}=0.755 \pm 0.08$

$E(B-V)=0.24 \pm 0.03$.

Our best estimate of the reddening is then $E(B-V)=0.24 \pm$ 0.03 and the best estimate of the distance to IC 4499 is $(m-M)_{0}=16.555-0.11 \times 0.755=16.472 \pm 0.016$ where the error estimate is the formal fitting error only. The DIRBE reddening map (Schlegel et al. 1998) gives $E(B-V)=0.23$ at the position of IC 4499 suggesting that the reddening is completely dominated by galactic reddening.

In addition to the fitting error we add the error contributions from the $K$-band zero-point $(0.025 \mathrm{mag})$, from the aperture correction $(0.02 \mathrm{mag})$, from the uncertainty in $A_{K}(0.009 \mathrm{mag})$, and from the uncertainty in the metallicity estimate $(0.231 \times$ $0.1=0.023 \mathrm{mag})$. This gives a final random error of $0.04 \mathrm{mag}$. The zero-point of the PL relation in Eq. (2) has an uncertainty of 0.044 mag which should be considered as a systematic error. We should also keep in mind that the metallicity scale is not uniquely defined and that there might be systematic differences between scales reaching 0.2 dex (Layden 1994). This would constitute a systematic error of $0.231 \times$ $0.2=0.046 \mathrm{mag}$. This adds up to an overall systematic error estimate of $0.06 \mathrm{mag}$. A much larger potential systematic error arises from the adopted zero-point of the $\left\langle M_{K}\right\rangle-\log P$ - $[\mathrm{Fe} / \mathrm{H}] \mathrm{re}-$ lation. As an example, Jones et al. (1992) found a zero-point based on Baade-Wesselink analysis of field RR Lyrae stars which is about 0.3 mag fainter than the B03 relation. A similar result was found by Layden et al. (1996) from a statistical parallax study of field RR Lyrae stars. Walker (1992) showed that adopting the Baade-Wesselink and statistical parallax zeropoint would cause the LMC distance estimate from RR Lyrae stars to be about 0.3 mag shorter than the canonical Cepheid based distance of $18.5 \mathrm{mag}$. This shows that the $\mathrm{B} 03$ relation is 
in good agreement with the canonical Cepheid distance scale but it disagrees with the short distance scale. B03 also show that their relation is in excellent agreement with the HST parallax measurements of RR Lyr itself (Benedict et al. 2002). Kovács (2003) has redone the Baade-Wesselink analysis for a sample of galactic Cepheids similar to the one of Jones et al. (1992) using the same calibration as has been successfully used for Cepheids and he now finds agreement with the canonical LMC distance. We cannot explain the difference between the distance scales with the current data, but we simply choose to adopt the B03 zero-point to ensure that we are on the canonical Cepheid distance scale.

On this basis we find $(m-M)_{0}=16.47 \pm 0.04($ random $) \pm$ 0.06 (systematic), or $d=19.7 \pm 0.4 \pm 0.6 \mathrm{kpc}$, as our best distance estimate to IC 4499 , which is in excellent agreement with the value of $(m-M)_{0}=16.45 \pm 0.05$ found by WN96. Our reddening value of $E(B-V)=0.24 \pm 0.03$ also agrees well with the value of $E(B-V)=0.22 \pm 0.02$ from WN96.

We stress that for stellar systems containing a reasonable number of RR Lyrae stars with known periods and metallicities, the $\left\langle M_{K}\right\rangle-\log P-[\mathrm{Fe} / \mathrm{H}]$ relation combined with single epoch $K$-band data gives accurate and reddening insensitive distances for a very modest amount of observational effort as the dominating source of error is not the number of individual phase points.

Acknowledgements. The data presented here was acquired during Directors Discretionary Time, granting of which is gratefully acknowledged. Thanks is due to Alistair Walker for providing a preprint of WN96 prior to publication, which helped the identification of the stars very much. Thanks is also due to Giuseppe Bono for comments on an early version of this paper and to an anonymous referee for suggestions which clarified the presentation.

\section{References}

van Albada, T. S., \& Baker, N. 1971, ApJ, 169, 311

Benedict, G. F., McArthur, B. E., Fredrick, L. W., et al. 2002, AJ, 123, 473
Bono, G., Caputo, F., Castellani, V., Marconi, M., \& Storm, J. 2001, MNRAS, 326, 1183

Bono, G., Caputo, F., Castellani, V., et al. 2003, MNRAS, 344, 1097 (B03)

Butler, D. J. 2003, A\&A, 405, 891

Cannon, R. D. 1992, unpublished, See Sarajedini 1993

Cardelli, J. A., Clayton, G. C., \& Mathis, J. S. 1989, ApJ, 345, 245

Carter, B. S. 1993, in Precision Photometry, ed. D. Kilkenny, E. Lastovica, \& J. W. Menzies, SAAO

Carter, B. S., \& Meadows, V. S. 1995, MNRAS, 276, 734

Castellani, M., Caputo, F., \& Castellani, V. 2003, A\&A, 410, 871

Clement, C. C., Dickens, R. J., \& Bingham, E. E. 1979, AJ, 84, 217

Clement, C. M., Nemec, J. M., Robert, N., et al. 1986, AJ, 92, 825

Coutts, C., Dickens, R. J., Epps, E., \& Read, M. 1975, ApJ, 197, L45

Ferraro, I., Ferraro, F. R., Fusi Pecci, F., Corsi, C. E., \& Buonanno, R. 1995, MNRAS, 275, 1057

Fourcade, C. R., \& Laborde, J. R. 1969, Mem. Soc. Astron. It., 40, 1

Fourcade, C. R., Laborde, J. R., \& Arias, J. C. 1974, A\&AS, 18, 3

Fusi Pecci, F., Bellazzini, M., Cacciari, C., \& Ferraro, F. R. 1995, AJ, 110,1664

Jones, R. V., Carney, B. W., \& Fulbright, J. P. 1996, PASP, 108, 877

Jones, R. V., Carney, B. W., Storm, J., \& Latham, D. W. 1992, ApJ, 386,646

Kovács, G. 2003, MNRAS, 342, L58

Layden, A. 1994, AJ, 108, 1016

Layden, A. C., Hanson, R. B., Hawley, S. L., Klemola, A. R., \& Hanley, C. J. 1996, AJ, 112, 2110

Lee, J.-W., \& Carney, B. W. 1999, AJ, 118, 1373

Lidman, C. 1995, IRAC-2B Test Rep. II, ESO

Longmore, A. J., Dixon, R., Skillen, I., Jameson, R. F., \& Fernley, J. A. 1990, MNRAS, 247, 684

Longmore, A. J., Fernley, J. A., \& Jameson, R. F. 1986, MNRAS, 220, 279

Moorwood, A., Finger, G., Biereichel, P., et al. 1992, The Messenger, 69,61

Sarajedini, A. 1993, AJ, 105, 2172

Schlegel, D. J., Finkbeiner, D. P., \& Davis, M. 1998, ApJ, 500, 525

Stetson, P. B. 1987, PASP, 99, 191

Suntzeff, N. B., Kinman, T. D., \& Kraft, R. P. 1991, ApJ, 367, 528

van den Bergh, S. 1993, MNRAS, 262, 588

Walker, A. R. 1992, ApJ, 390, L81

Walker, A. R., \& Nemec, J. M. 1996, AJ, 112, 2026 (WN96) 